Supporting information for $J A 038345 F$ :

\title{
A paramagnetic CEST Agent for imaging glucose by MRI
}

\author{
Shanrong Zhang, ${ }^{\#}$ Robert Trokowski ${ }^{\&}$ and A. Dean Sherry, ${ }^{\#,, *}$ \\ \# The Rogers Magnetic Resonance Center, Department of Radiology, University of Texas \\ Southwestern Medical Center, 5323 Harry Hines Blvd, Dallas, TX 75390-9085 \\ ${ }^{\&}$ Department of Chemistry, the University of Texas at Dallas, P.O. Box 830688, \\ Richardson, TX 75083-0688
}

* To whom the corresponding should be addressed

Email: sherry@utdallas.edu

Phone: 972-883-2907

Fax: $972-883-2925$ 
Figure s1. CD spectra of $\operatorname{Eu}(\mathbf{1})$ in presence of various amount of glucose in phosphate buffor at $\mathrm{pH}$ 7.2. And the peak intensities $(243 \mathrm{~nm})$ versus glucose concentration. By fitting to a 1:1 binding model, an association constant was obtained $\left(K a=377 \mathrm{M}^{-1}\right)$.
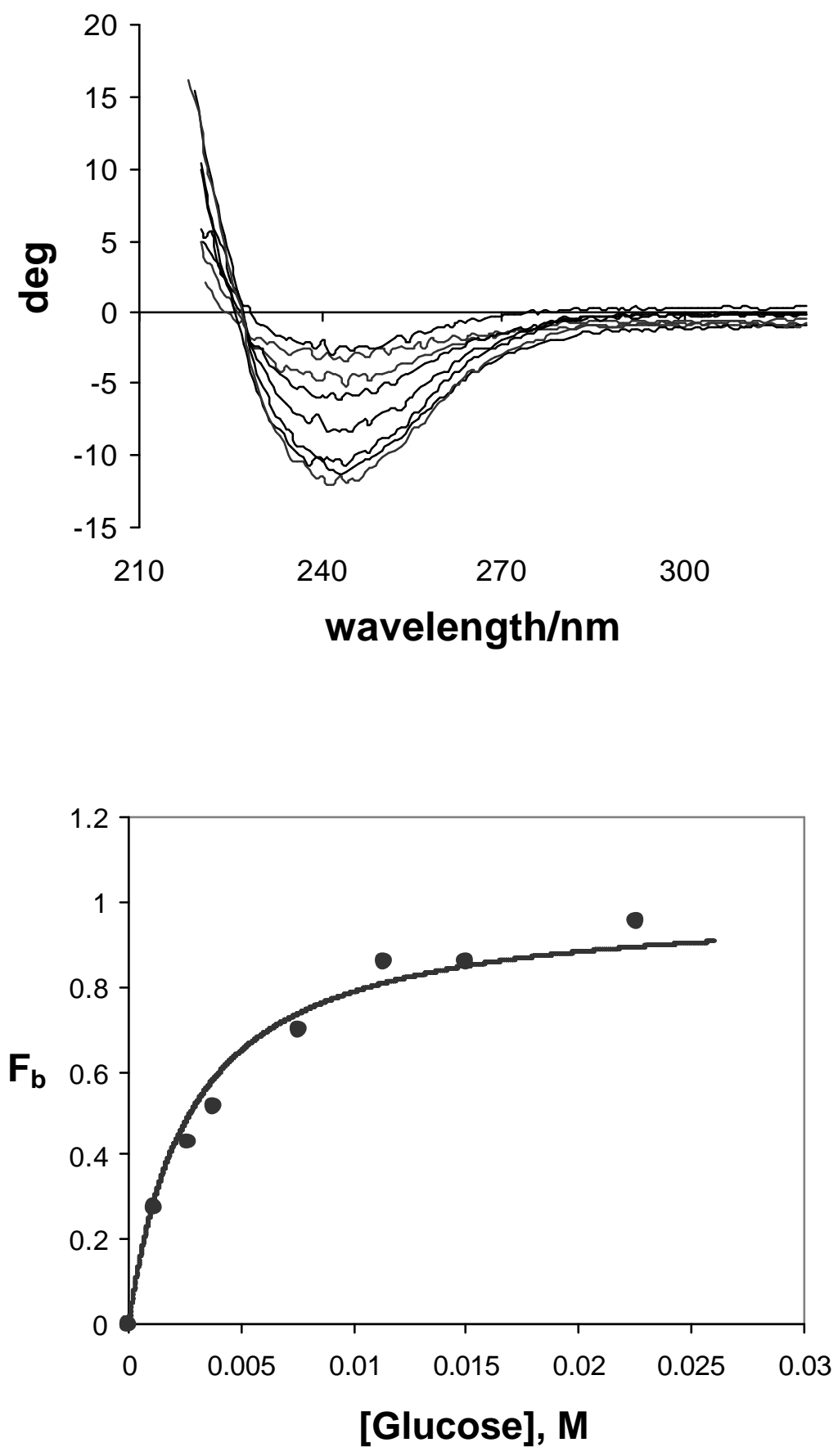
Figure s2. A plot of CEST subtraction versus glucose concentration for $10 \mathrm{mM} \operatorname{Eu}(\mathbf{1})$ complex in $100 \mathrm{mM}$ PIPES buffer at $\mathrm{pH} 7.0,25^{\circ} \mathrm{C}$. The CEST ratios were calculated from the corresponding CEST spectra by applying a $2 \mathrm{~s}$ saturation pulse $\left(\mathrm{B}_{1}=1020 \mathrm{~Hz}\right)$ at a frequency offsets of 50 and $30 \mathrm{ppm}$. The data points and error bars are the average values and standard deviations from three individual experiments. The solid curve is the best fitting to the experimental data, which gave $K a=383 \mathrm{M}^{-1}$.

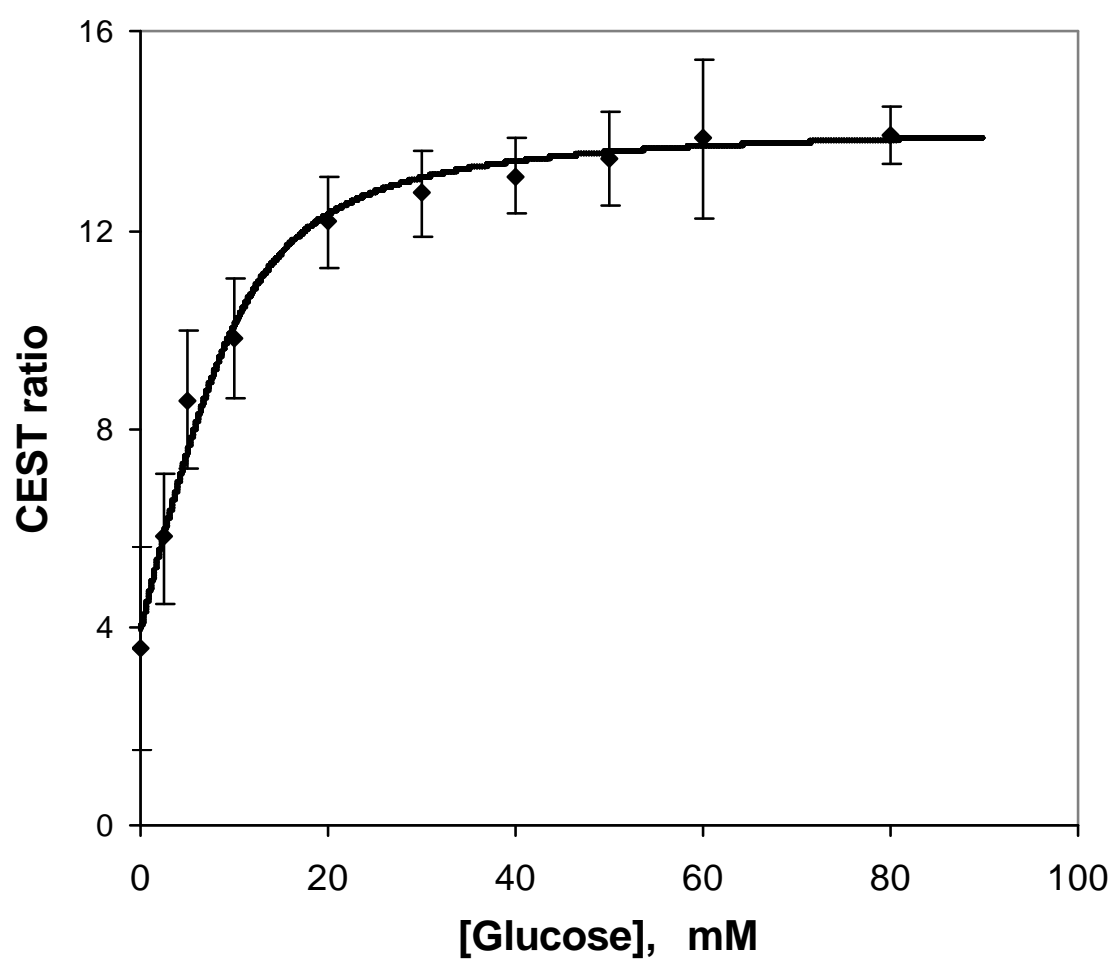


Figure s3. CEST image of a phantom consisting of four plastic tubes, each containing 10 $\mathrm{mM} \mathrm{Eu}(\mathbf{1})$ complex in $100 \mathrm{mM}$ HIPES buffer at $\mathrm{pH} 7.0$ and various amount of glucose, $0,5,10$ and $20 \mathrm{mM}$, respectively. The image No. 1, 2 and 3 are corresponding to that by applying a saturation pulse of $2 \mathrm{~s}$ at a power level of $36 \mathrm{db}\left(\mathrm{B}_{1}=1020 \mathrm{~Hz}\right)$ at a frequency offset of 50, 30 and $-50 \mathrm{ppm}$, respectively (The small dark dots at the edge are due to the micro bubbles sticking onto the surface of plastic tubes.).

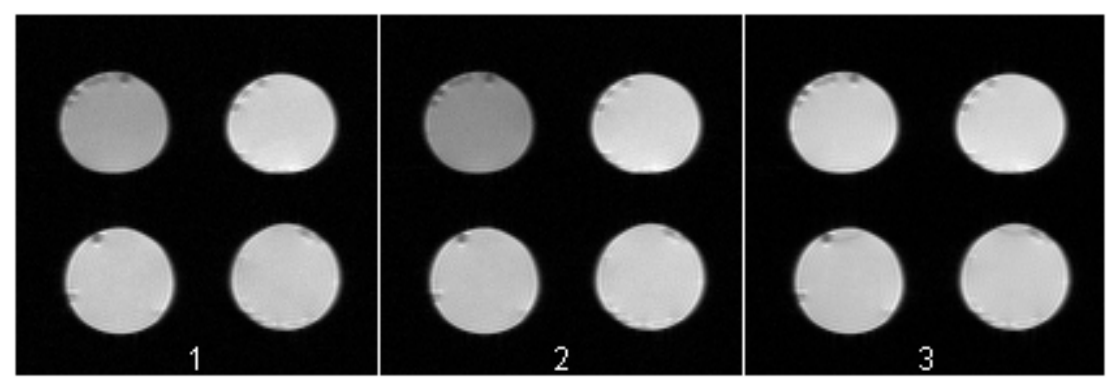

